\title{
Supporting Information: \\ Investigation of Adhesion Between Heavy Oil/Bitumen and Reservoir Rock: A Molecular Dynamics Study
}

\author{
Bablu Meghwal, Nakul Rampal ${ }^{1}$ and Ateeque Malani ${ }^{2}$
}

Department of Chemical Engineering, Indian Institute of Technology Bombay, Mumbai, India

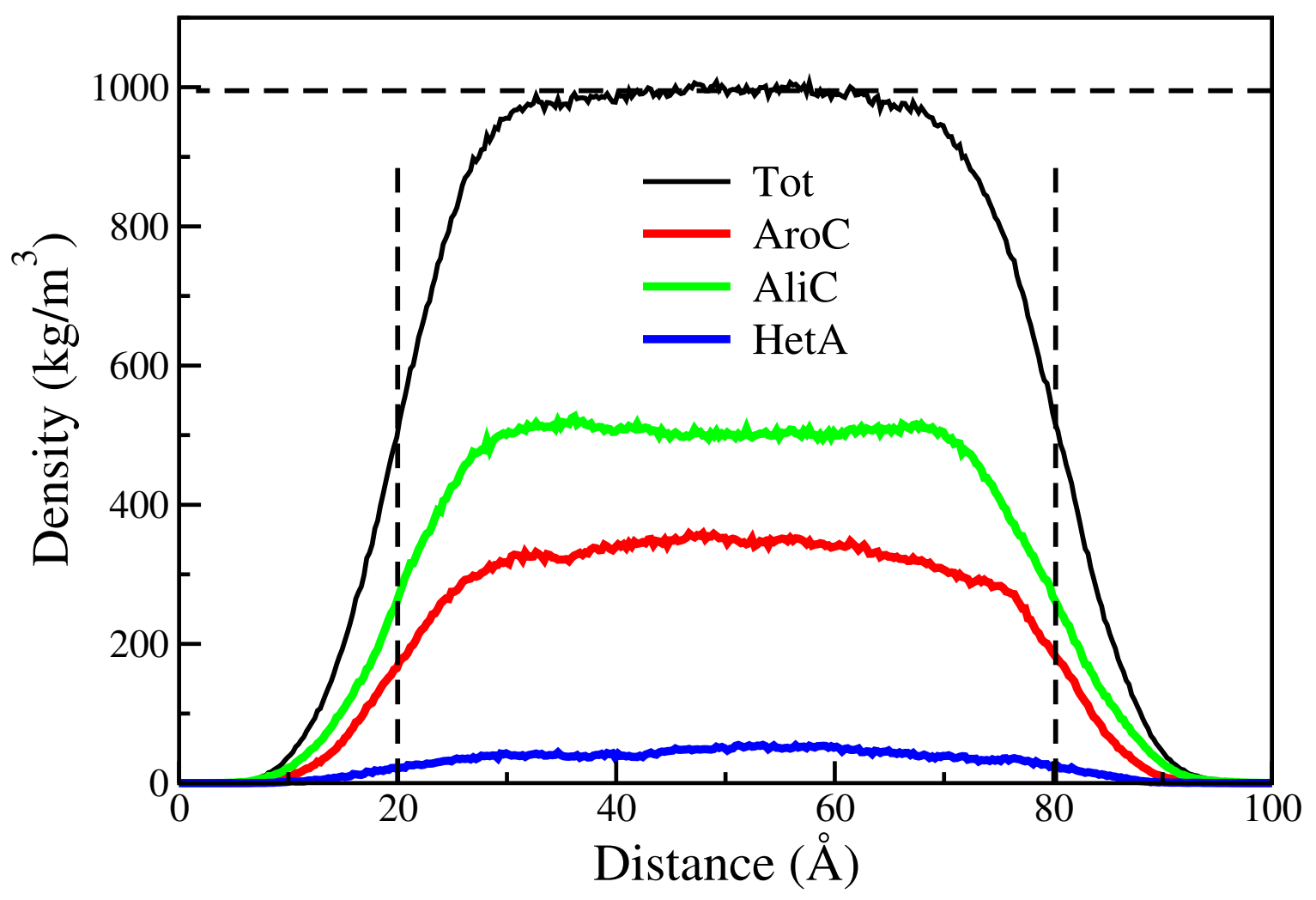

Figure S1: Density profile of heavy-oil slab along the $z$-direction obtained from the molecular dynamics (MD) simulations of 20 ns. The black line represent the density of heavy-oil (total) whereas red, green and blue lines represent mass density of aromatic carbons (AroC), aliphatic carbons (AliC) and heteroatoms (HetA), respectively. The mass density of heavy-oil is around $995 \mathrm{~kg} / \mathrm{m}^{3}$ (horizontal dashed line) which is in agreement with the data of Li and Greenfield ${ }^{1}$. The vertical two lines represent the position of liquid-vapor interface obtained by Gibbs dividing surface methodology ${ }^{2}$ The thickness of heavy-oil slab between these two interface is around 60 $\AA$.

${ }^{1}$ Current address: Adsorption and Advanced Materials Laboratory (AAML), Department of Chemical Engineering \& Biotechnology, University of Cambridge, Cambridge, CB3 0AS, UK

${ }^{2}$ Corresponding Author: amalani@iitb.ac.in 


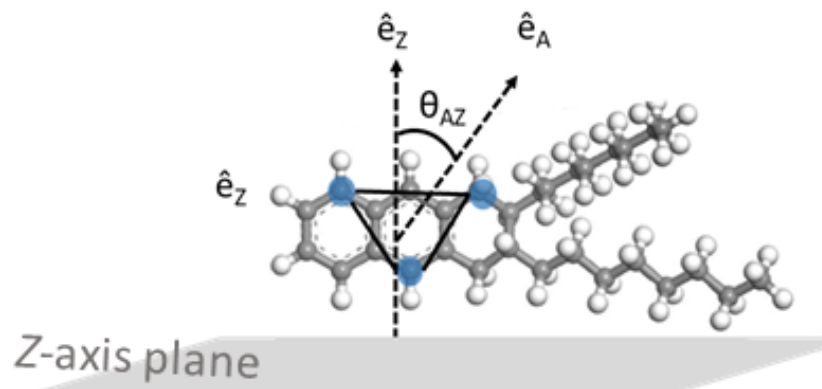

Ar2

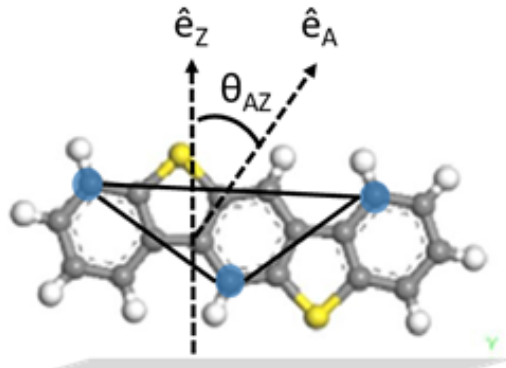

R3

$$
\theta_{A Z}=\cos ^{-1}\left(\hat{\mathrm{e}}_{A} \cdot \hat{\mathrm{e}}_{Z}\right)
$$

Figure S2: Schematic illustrating the orientational distribution of of dioctyl-cyclohexanenapthalene (DOCHN, Ar2) and Benzobisbenzothiophene (R3) molecule with respect to normal of the surface. The angle, $\theta_{A Z}=\cos \left(\hat{\mathrm{e}}_{A} \cdot \hat{\mathrm{e}}_{z}\right)$, is evaluated as the angle formed between, $\hat{\mathrm{e}}_{A}$, vector normal to aromatic plane of oil molecules (blue dots form an aromatic plane) and surface normal (i.e. $z$-axis, $\hat{\mathrm{e}}_{z}$ ). 


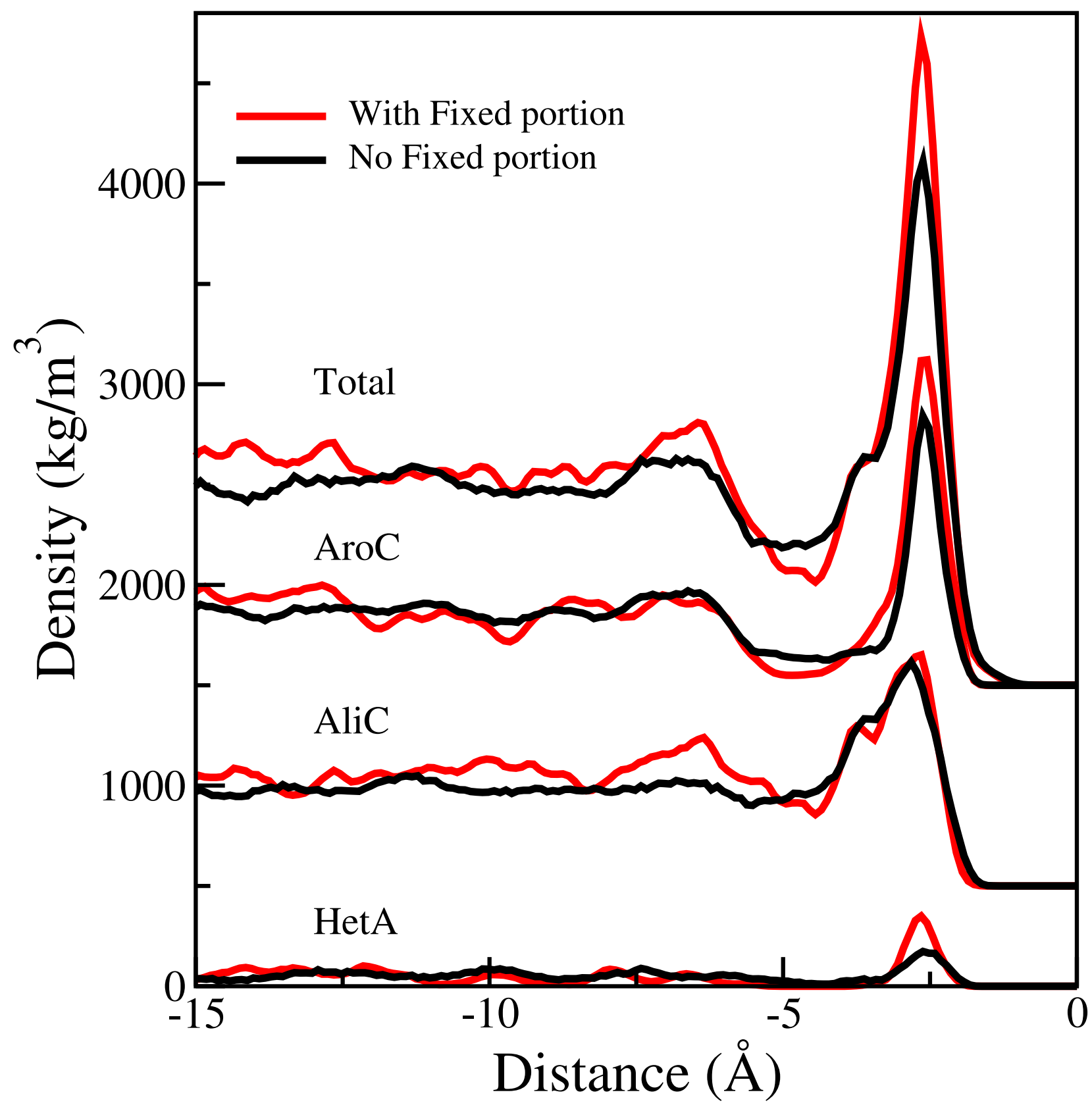

Figure S3: Density profiles of heavy oil (total) and its various components (AroC, AliC and HetA) adjacent to calcite surface at contact position (i.e. $\zeta=0$ ). The exposed atoms of calicate surface are present at reference (i.e. $z=0$ ). The density profiles are displaced along $y$-axis for clarity. The comparison is shown for two different sets of simulations containing half portion of heavy oil fixed (red lines) and all free portion of heavy oil (black lines). The excellent comparison between the two system indicates that the methodology of keeping some portion of heavy oil fixed to study adhesion does not affect the results. 

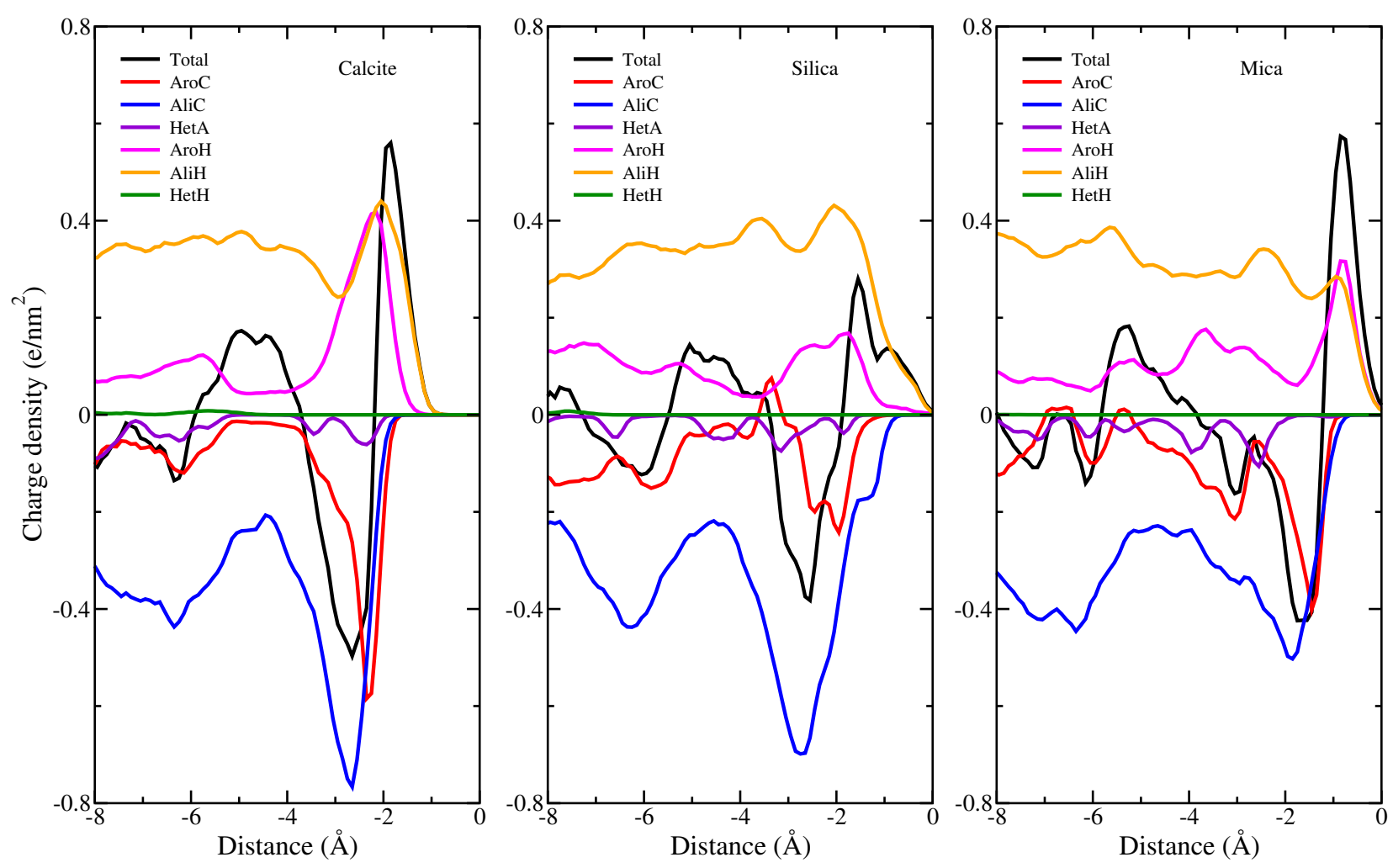

Figure S4: Charge density of heavy oil (total) and contribution from indivdual atom types (AroC - aromatic carbons, AliC - aliphatic carbons, HetA - heteroatoms, AroH - aromatic hydrogens, AliH - aliphatic hydrogens and HetH - hydrogen attached to heteroatoms) adjacent to rock surface. 


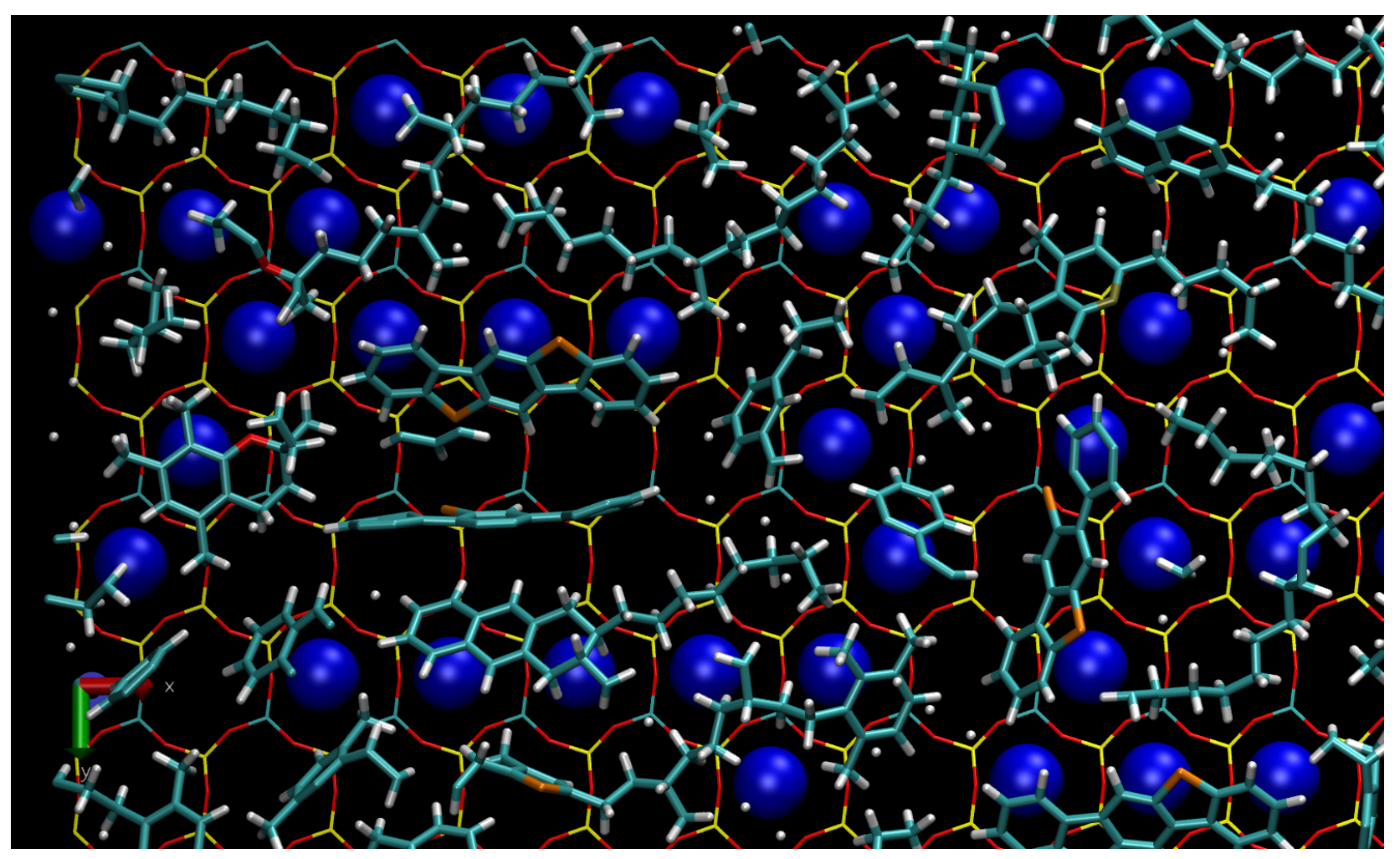

Figure S5: Snapshot of heavy oil-mica system at contact, $\zeta=0$ (only small prtion of the system is shown). The atoms within the interfacial layer of heavy oil and tetrahedral layers of mica surfaces are only shown for clarity. The mica surface atoms, except potassium ion (blue sphere) is shown in line format (Yellow - silica, Red - oxygen, Cyan - aluminium). Heavy oil atoms are shown in licorice (Cyan - carbon, White - hydrogen, Orange - sulphur). Orientation of heavy oil molecules is not parallel, rather inclined due to presence of surface potassium ions. 


\section{References}

[1] Li, D. D.; Greenfield, M. L. Chemical compositions of improved model asphalt systems for molecular simulations. Fuel 2014, 115, 347-356.

[2] Davis, H. T. Statistical Mechanics of Phases, Interfaces and Thin Films; VCH Publishers Inc.: New York, USA, 1996. 